\title{
Estudio de las Prácticas Culturales, Porosidad del Suelo y Gestión Hídrica en el Combate contra la Desertificación
}

Elsa P. Sampaio

Universidad de Évora, Departamento de Geociencias, Colégio Luís António Verney, Rua Romão Ramalho 59, 7000 Évora-Portugal (e-mail: ems@uevora.pt)

\begin{abstract}
Resumen
Se realizó un estudio comparativo entre un sistema de producción de cereales de secano de conservación y otro tradicional, con el fin de evaluar los efectos a largo plazo de diferentes prácticas culturales en el combate a la desertificación. El sistema de conservación ha recurrido a siembras directas a lo largo de 10 años consecutivos, mientras que el tradicional al laboreo del suelo. En un suelo tipo Chromic Vertisol en Portugal se evaluó a partir de imágenes en 2D, la continuidad del espacio poroso y, por tanto, la dinámica del agua del suelo. Para tener una buena representatividad de la zona bajo estudio, se analizaron muestras de $250 \mathrm{~cm}^{2}$ de suelo. Los resultados muestran que el sistema conservador, induce un aumento de la continuidad del espacio poroso en profundidad. Así, las prácticas culturales conservadoras, producen alteraciones en el régimen hídrico del suelo, siendo una importante estrategia a adoptar, como factor que interviene en el ciclo hidrológico y como acción concreta de lucha contra la desertificación.
\end{abstract}

Palabras clave: suelo, prácticas culturales, siembra directa, régimen hídrico, desertificación

\section{Cultural Practices Study, Soil Porosity and Water Management against Desertification Combat}

\begin{abstract}
To evaluate the long term effects of different cultural practices in the desertification combat, a comparative study between a dryland conservative cropping system of cereals and another more traditional one was done. The conservative system has had direct seeding along 10 consecutive years, while the traditional one has had soil tillage. On a soil of the type Chromic Vertisol in Portugal the continuity of the porous space and therefore the water dynamics of the soil were evaluated using 2D images. To have a better representation of the area under study, soil samples of $250 \mathrm{~cm}^{2}$ were analyzed. The results, demonstrated that the conservative system, induces an increase of the porous space continuity in depth. Thus, the cultural conservative practices produce alterations in the water regime of the soil, being an important strategy to adopt, as intervening factor in the hydrologic cycle and as a concrete action against desertification.
\end{abstract}

Keywords: soil, cultural practices, direct seed, water regime, desertification 


\section{INTRODUCCIÓN}

En un contexto de escasez y al mismo tiempo de aumento de búsqueda, la gestión de los recursos hídricos adquiere cada vez mayor importancia. De acuerdo con Goyal (2004), las alteraciones climáticas tendrán graves consecuencias en el ciclo hidrológico, principalmente, en la precipitación, en la evapotranspiración y en la humedad del suelo, entre otros. La desertificación, por ejemplo, sucede en ecosistemas de regiones secas o semiáridas, extremadamente vulnerables al sobreexplotación y al uso inadecuado del suelo y de su agua. Por otro lado, los riesgos de inundación son también resultado de estrategias inadecuadas de gestión del suelo.

Además, el régimen hídrico es uno de los factores clave que intervienen en las emisiones de óxido nitroso $\left(\mathrm{N}_{2} \mathrm{O}\right)$ por el suelo (Huang et al., 2007). La lixiviación del nitrógeno del suelo, puede ser una fuente potencial significativa de estas emisiones, comparable con el $\mathrm{N}_{2} \mathrm{O}$ emitido en sistemas convencionales de siembra (Lin et al., 2008).

Los ecosistemas agrícolas en regiones semiáridas, se caracterizan por lluvias erráticas e índices de alta evaporación, llevando a las producciones agrícolas a situaciones desfavorables. El total de lluvias estacionales puede ser suficiente para mantener la producción de cereales, pero su distribución y frecuencia de periodos de escasez, tanto dentro de la estación como entre estaciones, afectan a la producción (Stephen et al., 2005). Así, la estabilidad temporal del porcentaje de humedad del suelo puede tener profundas implicaciones en la agricultura en general, y en la gestión del agua en particular. Puede decirse que el conocimiento de la variabilidad espacio-temporal del agua del suelo, su estabilidad y distribución, puede constituir una base útil para una mejor gestión del agua (Starr, 2005).

En gran medida, las propiedades hidráulicas de los suelos son controladas por la geometría de las redes del espacio poroso del suelo, o sea, por su continuidad e tortuosidad. Vervoort et al. (2001), han adoptado la posición de Flury (1996), al afirmar que en sistemas no labrados, los flujos preferentes a través de macroporos estructurales y biológicos, pueden ser sustancialmente importantes en la profundidad alcanzada por la percolación del agua. Además Vervoort et al. (2001), indican que una buena investigación de la literatura, refiere que la cuantificación de la infiltración es complicada y que no existe un acuerdo consistente con respecto a la mejor técnica para evaluar diferentes mecanismos de infiltración, en diferentes sistemas de producción, con y sin laboreos.

Comprender los mecanismos del transporte y distribución del agua en suelos no saturados, mediante la red en tres dimensiones (3D) de su espacio poroso, ha sido de gran importancia y al mismo tiempo representa una dificultad para la ciencia. Según Weerts et al. (2001), la determinación de muchos de estos aspectos han sido el objetivo de varios trabajos tanto teóricos como experimentales, pero aún no son bien entendidos.

Muchos han sido los trabajos desarrollados en el sentido de cuantificar estas redes en 3D a partir de imágenes en 2D, a fin de prever el movimiento de los solutos (Banninger et al., 2006; Perret et al., 2000; Perret et al., 1999; Moreau et al., 1999; Anderson y Hopmans, 1994; McBratney et al., 1992; entre otros). Aún, si se trabajara con muestras demasiado pequeñas para una representación de la realidad de campo en "Vertisols" (FAO-UNESCO, 1988), que en la época seca y caliente abren hendiduras de mayor longitud y ancho que las propias muestras, no se ha llegado aún a conclusiones seguras, acerca de la propia metodología para utilizar estos objetivos, mucho menos, acerca de las relaciones entre las prácticas culturales y sus consecuencias en el régimen hídrico del suelo.

En Portugal la mayoría de los suelos se encuentra muy degradada. La principal causa de su degradación es la erosión provocada por la precipitación (el clima mediterráneo es caracterizado por distribución irregular de lluvia y ocurrencia de sequías). Generalmente la precipitación ocurre en la época más fría, o sea, en periodos poco vegetativos). Otras características de la mayoría de los suelos portugueses son, la baja o media capacidad de retención y de almacenamiento de agua, la baja o media fertilidad y bajos niveles de materia orgánica siendo en muchos casos, sujetos a escurrimientos superficiales. 
Así, el principal objetivo de este trabajo es contribuir para un mejor conocimiento de las condiciones necesarias para garantizar la existencia de agua y alimentos en los agro-sistemas de las zonas semiáridas de Europa, o sea, para disminuir la tendencia a la desertificación. Por condiciones necesarias, en este contexto, se consideran las prácticas culturales asociadas a los diferentes sistemas de producción de cereales de secano, muy tradicionales en el interior de las regiones mediterráneas de Europa, expresamente en Portugal. Se pretende evaluar las referidas condiciones a través de características hídricas del suelo, como por ejemplo mediante análisis del espacio poroso y de la dinámica de percolación del agua en profundidad.

\section{MATERIAL Y MÉTODOS}

El estudio se efectúo en un ensayo de producción de cereales de secano, ubicado en una unidad homogénea de terreno, con un declive del $2 \%$, en el sur de Portugal ( $37^{\circ} 59^{\prime} \mathrm{N}$ : $7^{\circ} 57^{\prime} \mathrm{W}$ ), con clima mediterráneo, en un Chromic Vertisol, de acuerdo con la World Reference Base for Soil Resources WRBSR, de la IUSS Working Group WRB (2006), típico de las condiciones edafoclimáticas mediterráneas.

La nomenclatura de horizontes y capas de suelo utilizada en el trabajo aquí presentado es de la propuesta por Soil Survey Staff (SSS, 1981).

En esta unidad homogénea de Chromic Vertisol, está instalada una rotación de cultivos tradicionales de secano, típica de la región (Girasol - Trigo Blando - Cebada Dística).

En una sub-unidad de terreno los cultivos están sometidos, desde hace más de 50 años, al sistema de producción tradicional, con ayuda de las técnicas de movilización de suelo tradicionales (ST). En otra sub-unidad, se respetó los mismos cultivos, bajo condiciones de un sistema de producción de conservación que en los últimos 10 años consecutivos, recibió un conjunto de prácticas agrícolas que utilizaban las siembras directas (SD10).

En el sistema tradicional o convencional (ST) se llevaron a cabo las siguientes prácticas agrícolas: labranzas de $30 \mathrm{~cm}$ de profundidad para el control de plantas espontáneas; labradas a $30 \mathrm{~cm}$ de profundidad y gradeos cruzados para preparar la capa de la semillas; siembras; rastrillajes; mondaderas mecánicas; tratamientos fitosanitarios y abonos. En el sistema de conservación con siembra directa (SD10) se llevaron a cabo las siguientes prácticas agrícolas: control de plantas espontáneas en la pre-siembra con un herbicida total sistémico no residual; siembra directa y abonos.

La época del año en que se han desarrollado trabajos de campo ha sido en Octubre y Noviembre, lo que corresponde a la época de siembra con el suelo a iniciar su fase de humedecimiento y expansión, que cierra los espacios abiertos durante el verano. Espacios es un término equivalente a poros, pero es preferido en esto trabajo porque, de acuerdo con Warkentin (2008), no tiene conotacion con cualquier forma.

La continuidad de la red del espacio poroso fue evaluada mediante el método de infiltración de yeso en el perfil del suelo (Bouma et al., 1982). Después del secado y endurecimiento del yeso dentro del espacio poroso del suelo, fueron cortadas tajadas de superficies de suelo de 10 en $10 \mathrm{~cm}$ de profundidad. En cada una de las superficies expuestas, los poros llenos de yeso, fueron identificados y contabilizados por formas y clases de dimensión. Se ha optado por el yeso por ser un material poco degradante para los suelos y dar resultados suficientemente fiables (Bouma et al., 1982). Los aspectos relacionados con la semejanza entre la viscosidad del agua de lluvia y de la suspensión con yeso $u$ otros materiales, no son considerados influyentes en la degradación del suelo, en comparación con los resultados obtenidos (McBratney et al., 1992). El yeso ha sido coloreado de azul para no ser confundido con materiales constituyentes del suelo.

El primer criterio de diferenciación para los vacíos fueron: con yeso y sin yeso, correspondientes a continuo y no continuo. Se contabilizaron los vacíos continuos y la diferencia entre estos y la porosidad total puede dar el valor de los no continuos sin que exista necesidad de diferenciarlos y contarlos de uno a uno. La opción por este método, aparentemente anticuado, se justifica al hecho 
de muchos métodos modernos de estudio en 3D de este espacio (Ligthart et al., 1993), sea por difusión de gas (Moldrup et al., 2001; Inversen et al., 2001; Poulsen et al., 2001), sea por tomografia axial computarizada -TAC (Warner et al., 1989; Perret et al., 2000), sea por los modelos de simulación de Lattice-Boltzmann (Torquato et al., 1999), sea por geometria fractal (Anderson et al., 2000; Filgueira et al., 1999; Bird et al., 2000), u otros, no tienen datos resultados suficientemente próximos de las características del espacio de cultivo. Así, aún existe la necesidad de mejorar considerablemente aquellos métodos (Weerts et al., 2001).

Enseguida se diferencian: hendiduras $(F)$, con por lo menos el doble de la longitud en relación al ancho; bioporos $(P)$ redondos o ligeramente ovales debido a la oblicuidad de los canales formados por ellos en relación a los cortes horizontales de observación; espacios irregulares (Esp), de formatos diversos. Cada uno de estos se diferencian después de acuerdo con sus dimensiones teniendo en cuenta la influencia que ejercen sobre el drenaje, el almacenamiento de agua y la producción de cereales, de acuerdo con Goss et al. (1978).

En cada uno de los sistemas de producción, se han seleccionado, al azar, 4 bloques (repeticiones), para ejecutar los trabajos de preparación del suelo y recogida de imágenes para evaluación del espacio poroso, en un total de 8 perfiles. Cada superficie trabajada es un cuadrado de ochenta centímetros de lado, de los cuales, el cuadrado central con cincuenta centímetros de lado (2500 $\mathrm{cm}^{2}$ ), constituye la superficie de análisis. Esta ha sido la medida de la entrelínea de las sembradoras utilizadas en ambos los sistemas, y así toda la variedad posible de características del espacio de cultivo ha sido evaluada. La utilización de una superficie de $80 \mathrm{~cm}$ de lado para analizar el cuadrado central con $50 \mathrm{~cm}$ de lado, fue para compensar, en la contabilización en profundidad, los poros oblicuos que salen del área en estudio por los que se dejan infiltrar en los $30 \mathrm{~cm}$ periféricos superficiales pero surgen en el centro, en profundidad.

En cada una de las 8 superficies de trabajo (4 por cada sistema de producción), se hizo una imagen correspondiente al cuadrado central con $50 \mathrm{~cm}$ de lado, de 10 en $10 \mathrm{~cm}$ de profundidad, hasta dejar de verse cualquier vacío coloreado de yeso azul. Las imágenes de cada superficie de observación fueron recogidas con una cámara fotográfica. Se han obtenido así, imágenes digitales "True Color RGB", con 24 "bits" por "píxel", con una captura de 18 MB cada una.

Así, para el trabajo del campo se utiliza material muy simple y accesible (una moldura en hierro con $80 \times 80 \mathrm{~cm}$ de lado para permitir la impregnación del suelo con la suspensión de yeso sin que esta se esparza en superficie; yeso; agua; cuchillos y otros materiales cortantes de pequeñas dimensiones; escobas y aspiradores para limpiar las superficies; soplete pequeño para quemar residuos vegetales y secar las superficies; máquina fotográfica).

Según Chertkov y Ravina (1999), la estimación directa a partir de imágenes a dos dimensiones (2D), es bastante práctica para prever un número suficientemente grande de conexiones de la red. No se pretende reconstruir la red $3 \mathrm{D}$ a partir de imágenes $2 \mathrm{D}$, pero si, evaluar la funcionalidad de esa red 3D en su capacidad de percolación en profundidad.

El reconocimiento automático de las varias clases de poros mediante el color, se ha efectuado en un analizador de imágenes denominado "Sigma Scan Pro 5.0 Image Analysis Software" (SPSS, 1999), con las siguientes características básicas, entre otras: identificación cromática; mediciones morfométricas; mediciones de intensidad; hoja de cálculo interna; resultados gráficos; otras herramientas. Así se recurre a las operaciones clásicas de análisis de imágenes, con "Color Threshold". Dado que el yeso utilizado había sido coloreado de azul, fue efectuada una calibración de los límites de los valores del "Croma" y de la "Intensidad" correspondientes. Así, fueron seleccionados todos los "píxeles" con valores de Croma, identificado como "Hue", entre 132 y 203, y de Intensidad entre 0 y 100.

Se han contabilizado todos los elementos del espacio poroso, hasta la dimensión mínima posible, de acuerdo con los limites de las clases de dimensiones para el crecimiento de cereales, drenaje y almacenamiento de agua, definido por Goss et al. (1978). 
Así, las clases de dimensiones de bioporos contabilizados han sido: bioporos grandes PG $(\varnothing>5$ $\mathrm{mm})$; bioporos medios PM $(1<\varnothing \leq 5 \mathrm{~mm})$; bioporos pequeños $\mathrm{PP}(0,4<\varnothing \leq 1 \mathrm{~mm})$; bioporos muy pequeños $\operatorname{Pmp}(0,15<\varnothing \leq 0,4 \mathrm{~mm})$ y bioporos mínimos $\operatorname{Pm}(0,03<\varnothing \leq 0,15 \mathrm{~mm})$.

Las hendiduras han sido diferenciadas con base en las siguientes clases de dimensiones: hendiduras largas FL $\geq 200 \mathrm{~mm}$; hendiduras medias ( $<$ FM $\leq 200 \mathrm{~mm}$ ); hendiduras cortas (FC $\leq 5$ $\mathrm{mm}$ ). En una segunda fase, dentro de cada clase de longitud, han sido diferenciadas de acuerdo con el ancho en: anchas (anchura $\geq 5 \mathrm{~mm}$ ); medias $(1<$ anchura $\leq 5 \mathrm{~mm}$ ) y finas (anchura $\leq 1 \mathrm{~mm}$ ).

No se han diferenciado clases en los espacios irregulares, ya que no se ha encontrado ningún parámetro que pudiese servir a tal objetivo.

Para cada objeto identificado, fueron automáticamente medidos: área, perímetro, factor de circulación, diámetro de Feret, eje mayor y eje menor. En una hoja de cálculo interna se ha construido un "transform", o sea, un programa construido dentro de esa hoja con un lenguaje informático propio, que ha servido para separar los bioporos de los espacios irregulares de las hendiduras y sus respectivas clases de dimensiones. En este "transform" se ha calculado también, el área ocupada por el total de los bioporos de cada clase; área ocupada por el mayor y por el menor bioporo; el diámetro del bioporo menor encontrado; el número de bioporos encontrados; la media de las áreas; el desviación-patrón; el error-patrón y la relación centímetros cuadrados de bioporos por metro cuadrado de área analizada. El mismo conjunto de parámetros fue calculado para cada clase de hendiduras. En cuanto a los espacios irregulares, fue medido el área de cada uno y todos los restantes parámetros calculados en los otros dos casos.

Las referidas mediciones y cálculos fueron efectuados para cada una de las 4 superficies (perfiles repetidos en cada sistema de producción) a cada $10 \mathrm{~cm}$ de profundidad hasta los $80 \mathrm{~cm}$ de profundidad en cada sistema de producción, en un total de 72 imágenes analizadas,.

Los datos obtenidos fueron procesados en el software estadístico SPSS (SPSS, 2007), mediante análisis de varianza y valores de las medias de cada tipo y clase de poro, en cuadruplicados, para cada profundidad de cada sistema de producción. Fueron también evaluadas las correlaciones de Pearson $(r)$, y respectiva significancia $(p)$, para analizar las relaciones de cada tipo y clase de poro, a cada profundidad, entre los diversos perfiles.

\section{RESULTADOS Y DISCUSIÓN}

Con el fin de observar la evolución en profundidad de los bioporos, hendiduras y espacios irregulares, continuos, o sea, rellenados con yeso desde la superficie, se organizan los datos obtenidos para cada profundidad, por tipo de sistema de producción (ST o SD10) y se ha establecido su secuencia en profundidad. Con estos datos se han construido gráficos con las áreas ocupadas por cada tipo y clase de dimensiones establecidas. Estos gráficos, presentados en las Fig. 1 y 2 , han sido construidos de forma para obtener las informaciones ya referidas en su relación con la profundidad y, aún, con la identificación de los horizontes o capas, de cada perfil. Los resultados se presentan en la forma del área ocupada por cada tipo y clase de dimensión por unidad de superficie $\left(\mathrm{cm}^{2} / \mathrm{m}^{2}\right)$, y por el número de objetos o unidades, por la unidad de superficie, y igualmente, por cada tipo y clase de dimensión.

Las correlaciones de Pearson ( $r$ ), y respectiva significancia $(p)$ de cada tipo y clase de poro, a cada profundidad entre los diversos perfiles $(n=4)$ de cada sistema de producción, han sido positivas, variando entre valores de $r=0,914$ y 0,891 y valores de $p<0,01$. Para cada tipo y clase de poro, a cada profundidad, entre perfiles de sistemas diferentes, la correlación no se ha mostrado significativa, variando entre $p>0,09$. Estos valores indican que las medias de las 4 repeticiones en cada sistema de producción, son representativas del comportamiento de cada tipo y clase de poro del respectivo sistema de producción. Así, los diversos resultados se presentan en relación a un perfil-tipo para cada sistema de producción. 


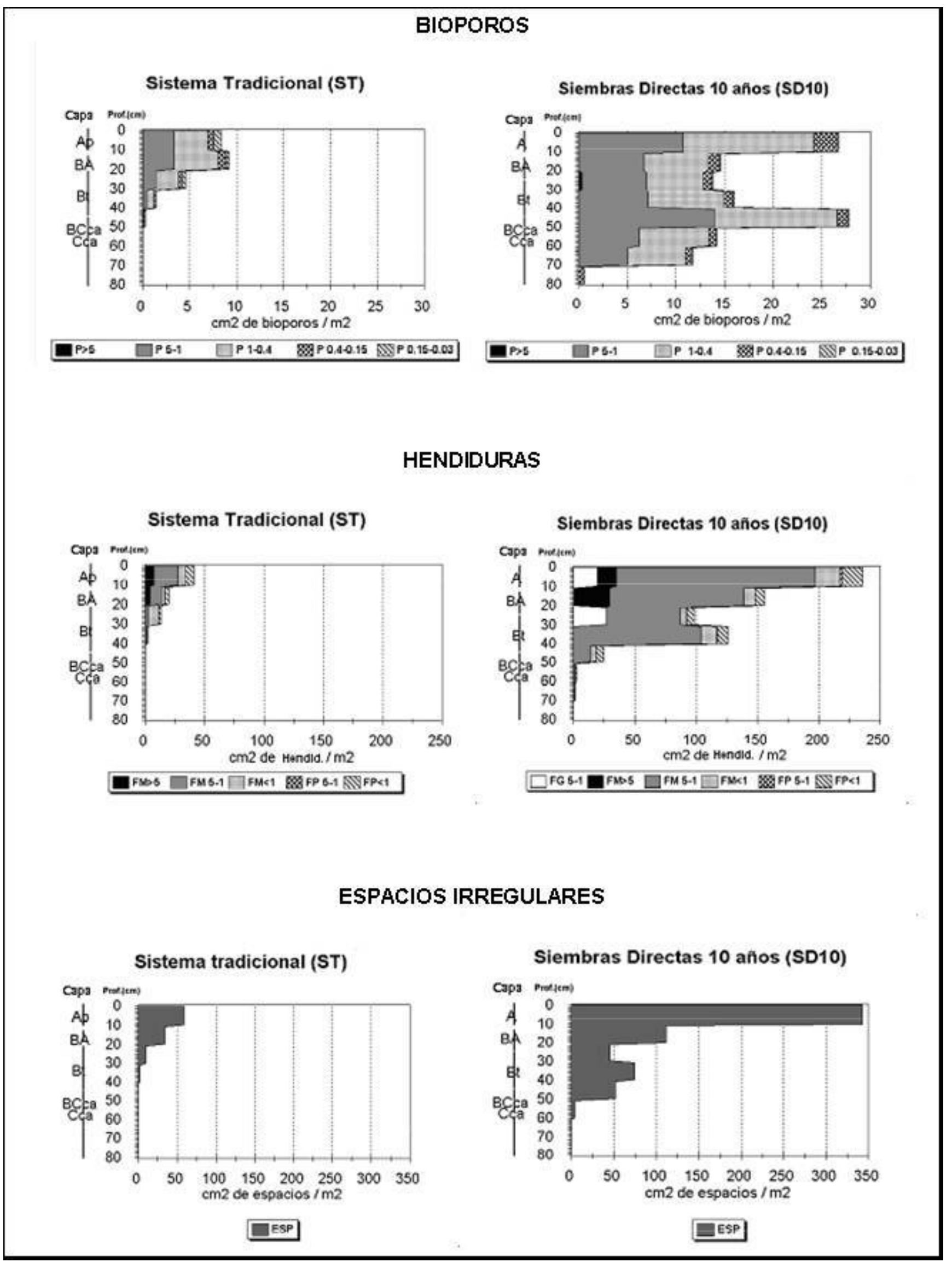

Fig.1: Continuidad de los parametros estudiados en $\mathrm{cm}^{2} / \mathrm{m}^{2}$ (ST/SD10) 


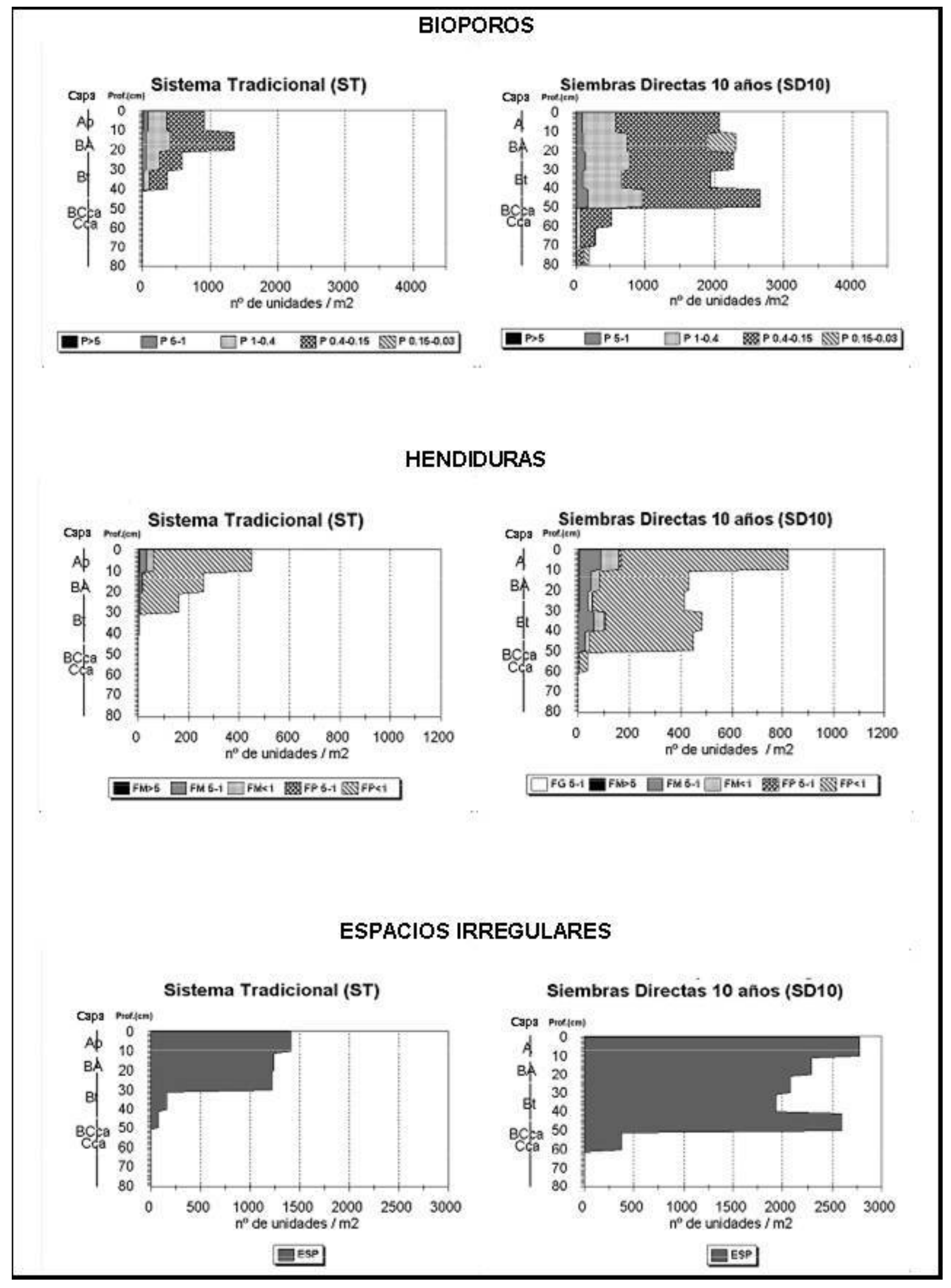

Fig.2: Continuidad de los parametros estudiados en $n^{\circ}$ de unidades $/ \mathrm{m}^{2}$ (ST/SD10) 
Los valores de $(r)$ y $(p)$ indican igualmente que se confirma que las diferencias encontradas en cada tipo y clase de poro, a cada profundidad, están relacionadas en la diferencia del sistema de producción.

Teniendo en atención el elevado grado de degradación la que los suelos están sujetos en los sistemas de producción de cereales de secano, tal como ya fue constatado por Jury y Vaux Jr. (2007), del análisis de la distribución de la porosidad por los tipos y clases de dimensiones en cada sistema, se destaca que:

- Tanto las F como los Esp ocupan siempre mayor área de que los P;

- En ST, las áreas ocupadas por cada tipo y clase de dimensión disminuyen progresivamente con la profundidad, en cuanto que en SD10 existe una reducción brusca por debajo de los $10 \mathrm{~cm}$ pero, desde ahí hacia abajo, esa reducción es progresiva y suave. Aun, en SD10 los P ocupan un área importante en profundidad existiendo asimismo un aumento en la parte inferior del horizonte $\mathrm{Bt}$;

- Las áreas continuas son significativamente mayores en SD10;

- La diferencia de la profundidad alcanzada por los espacios continuos también sufre aumentos de ST $(50 \mathrm{~cm})$ para SD10 $(60 \mathrm{~cm})$;

- En cuanto a las áreas ocupadas por cada clase de dimensión en ambos sistemas, en los $\mathrm{P}$, son las clases que varían entre los 5 y los $0,4 \mathrm{~mm}$ de diámetro las que más área ocupan, seguidas de los $0,15<P \leq 0,4$ en cuanto que los $P \geq 5$ y los $0,03<P \leq 0,15$ ocupan áreas muy reducidas o incluso inexistentes;

- En ST las áreas ocupadas por F, sean de las clases que sean, son insignificantes por debajo de los $30 \mathrm{~cm}(\mathrm{Bt})$;

- Las clases de $\mathrm{F}$ que ocupan mayores áreas, tal como con los $\mathrm{P}$, son las de dimensiones medias, comparativamente con las de gran o de pequeña dimensión. Aún así, la clase de $\mathrm{F}$ más pequeña tiene una presencia más significativa que la clase menor de $\mathrm{P}$;

- Las $\mathrm{F}$ de mayores dimensiones surgen en los horizontes superficiales, pero las de gran dimensión $(1<\mathrm{FG} \leq 5)$ surgen en SD10 entre la superficie y los $30 \mathrm{~cm}$ de profundidad;

- El número de unidades de cada clase de dimensión que más aumentó con el sistema SD fue, tanto en los bioporos como en las hendiduras, el de las clases de menor dimensión, a pesar de en términos de área ocupada, no han sido estas las clases que más han aumentado. Esto es, porque las clases de menor dimensión pueden aumentar su número de presencias unitarias, pero eso no repercute en un aumento proporcional de área, exactamente porque tienen pequeñas dimensiones;

- Una vez que son los bioporos de las clases de menor dimensión las que tienen mayor influencia en la capacidad de almacenamiento de agua en el suelo, lo que está de acuerdo con Alaoui y Helbling (2006), queda garantizado que los cultivos tienen un grado de humedad que satisfaga sus necesidades, igual ocurre cuando existen inestabilidades en los regimenes pluviométricos, o sea, se garantiza transponer la preocupación de Stephen et al. (2005), con una mayor estabilidad temporal del porcentaje de humedad del suelo;

- Es importante destacar que en las hendiduras, también son las clases de dimensión menor las que aumentan su número de presencias, y así se garantiza un transporte del agua en profundidad sin que este sea efectuado de una forma demasiado rápida para los horizontes mas profundos, con la consecuente lixiviación del nitrógeno del suelo, reduciendo, tal como fue estudiado por Huang et al. (2007) y Lin (2007), las emisiones de óxido nitroso por el suelo. Así, queda asegurado un menor riesgo de inundación y, a medida que el agua va penetrando en profundidad, se va teniendo oportunidades para comunicar con todos los horizontes intermedios por donde pasa. Así, siguiendo 
la lógica de Flury (1996), se garantiza la recarga de acuíferos, a través del suelo, con menor arrastre de materiales en profundidad, en forma simultánea con la reducción de los riesgos de erosión.

Todas estas mejorías en las caracteristicas de los suelos son consecuencia del aumento de la cantidad, tanto de bioporos, como de las hendiduras y espacios irregulares continuos, lo cual es directamente proporcional al número de años de SD. Este aumento se ha revelado también, en la profundidad de alcance de esa continuidad y por lo tanto, en las condiciones para producción de alimentos, bien como en la percolación de agua en profundidad con todas sus consecuencias en la recarga de acuíferos y reducción de los riesgos de erosión, o sea, satisfacen positivamente a los objetivos de este trabajo.

Todo esto viene a confirmar las conclusiones de Osunbitan et al. (2005), quienes afirman que la porosidad total no es la mayor determinante de la conductividade hidráulica en el suelo, debido a los disturbios que los sistemas de producción tradicionales provocan en la continuidad de los macroporos. Estos resultados vienen confirmar lo que se concluyó en relación a suelos en la India, donde segundo Sarkar y Singh (2007), la forma, la dimensión y la continuidad de los poros del suelo son determinados por las praticas culturales.

De este modo, las actuales preocupaciones con las crisis hídrica glogal y crisis de subsistencia alimentar que se aproximan (Jury y Vaux Jr., 2007; Lal, 2007 y Graham et al., 2007), disminuyen al analizar los resultados obtenidos en el sistema SD10.

Pero, estos resultados necesitan de ser complementados tal como ya Lal (2007) refirió, por estudios científicos más profundizados en términos de la gestión de los recursos suelo y agua, conjugados con las realidades sociales economicas y politicas. Solo así se podrán identificar los sistemas de producción y prácticas culturales, substitutos de aquellos que tradicionalmente son practicados, con todas sus consecuencias (Ryan et al., 2008).

\section{CONCLUSIONES}

Después del análisis y discusión de los resultados presentados, se puede concluir que en regiones interiores de Climas Mediterráneos en Europa, en un "Chromic Vertisol":

- El sistema de agricultura conservador que recurre a siembras directas a lo largo de periodos consecutivos prolongados, produce alteraciones benéficas en las características hídricas del suelo como factor que interviene en el ciclo hidrológico.

- Las características mejoradas por el sistema de conservación, permitieron que el suelo presentara un espacio poroso con una continuidad importante en profundidad y así la dinámica de percolación del agua en profundidad queda asegurada.

- La continuidad es garantizada sobre todo por vacíos de clases de menor dimensión y así el grado de humedad del suelo puede tener estabilidad temporal en profundidad y satisfacer las necesidades de la producción vegetal.

- Las alteraciones en el aumento de la eficacia del uso del agua del suelo, contribuyen a un aumento de la cantidad y calidad de las culturas para alimento y para la calidad de los propios suelos que resisten mejor a la erosión.

- La siembra directa, representa una importante alternativa para la reducción de la degradación medioambiental inducida por la agricultura, y ha demostrado ser una importante estrategia a adoptar en la gestión del suelo, como alternativa en el combate a la desertificación.

-Las prácticas culturales asociadas a los sistemas de producción conservadores, como el de la siembra directa, representan opciones claras en la lucha contra la desertificación, pero dejan claro que esta lucha necesita que la investigación futura las integre en las realidades sociales economicas y politicas. 


\section{REFERENCIAS}

Alaoui A. y A. Helbling; Evaluation of soil compaction using hydrodynamic water content variation: Comparison between compacted and non-compacted soil, Geoderma: 134 (2), 97-108 (2006).

Anderson S.H. y J.W. Hopmans; Tomography of soil-water-root processes, American Society of Agronomy, Soil Science Society of America, Madison Wisconsin, Minnesota, USA (1994).

Anderson A.N., J.W. Crawford y A.B. McBratney; On Diffusion in Fractal Soil Structures, Soil Science Society of America Journal: 64, 19-24 (2000).

Banninger D., P. Lehmann y H. Fluhler; Modelling the effect of particle size, shape and orientation of light transfer through porous media, European Journal of Soil Science: 57(6), 906-915 (2006).

Bird N.R.A., E. Perrier y M. Rieu; The water retention function for a model of soil structure with pore and solid fractal distributions, European Journal of Soil Science: 51, 55-63 (2000).

Bouma J., C.F.M. Belmans y L. W. Dekker; Water infiltration and redistribution in a Silt Loam subsoil with vertical worm channels, Soil Science Society of America Journal: 46, 917-921 (1982).

Chertkov V. . e I. Ravina; Tortuosity of crack networks in swelling clay soils, Soil Science Society of America Journal: 63, 1523-1530 (1999).

FAO - UNESCO; Soil map of the world 1:5 000 000. Revised Legend, World Soil Resources Report: 60 , Rome (1988).

Filgueira R.R. y otros cuatro autores; Comparison of fractal dimensions estimated from aggregate mass-size distribution and water retention scaling, Soil Science: 164, 217-223 (1999).

Flury M.; Experimental evidence of transport of pesticides through field soils - A review, Journal of Environmental and Quality: 25, 25-45 (1996).

Goss M.J., K.R. Howse y W. Harris; Effects of cultivation on soil water retention and water use by cereals in clay soils, Journal of Soil Science: 29, 475-488 (1978).

Goyal R.K.; Sensitivity of evapotranspiration to glogal warming: a case study of arid zone of Rajasthan (India), Agricultural Water Management: 69(1), 1-11 (2004).

Graham R.D. y otros dieciséis autores; Nutritious Subsistence Food Systems, Advances in Agronomy: 92, 1-74 (2007).

Huang S., H.K. Pant y J. Lu; Effects of water regimes on nitrous oxide emissions from soils, Ecological Engineering: 31(1), 9-15 (2007).

Inversen B.V., P. Schjonning, T.G. Poulsen y P. Moldrup; In situ, on-site and laboratory measurements of soil air permeability: Boundary conditions andmeasurement scale, Soil Science: 166, 97-106, (2001).

IUSS Working Group WRB; World Reference Base for Soil Resources 2006, $2^{\text {nd }}$ edition, World Soil Resources Reports: 103, FAO, Rome (2006).

Jury W.A. y H.J. Vaux Jr.; The Emerging Global Water Crisis: Managing Scarcity and Conflict Between Water Users; Advances in Agronomy: 95, 1-76 (2007).

Lal R.; Anthropogenic Influences on World Soils and Implications to Global Food Security, Advances in Agronomy: 93, 69-93 (2007). 
Ligthart T. N., G.J.W.C. Peek y E.J. Taber; A method for the three-dimensional mapping of earthworm burrow systems, Geoderma: 57, 129-141 (1993).

Lin L., C.Y. Lan, L.N. Huang y G.Y.S. Chan; Anthropogenic $\mathrm{N}_{2} \mathrm{O}$ production from landfill leachate treatment, Journal of Environmental Management: 87(3), 341-349 (2008).

McBratney A.B. y otros cuatro autores; Modifications to a method of rapid assement of soil macropore structure by image analysis, Geoderma: 53(3-4), 255-274 (1992).

Moldrup P. y otros cuatro autores; Tortuosity, diffusivity, and permeability in the soil liquid and gaseous phases, Soil Science Society of America Journal: 65, 613-623 (2001).

Moreau E., B. Velde y F. Terribile; Comparison of $2 D$ and $3 D$ images of fractures in a Vertisol, Geoderma: 92, 55-72 (1999).

Osunbitan J.A., D.J. Oyedele y K.O. Adekalu; Tillage effects on bulk density, hydraulic conductivity and strength of a loamy sand soil in southwestern Nigeria, Soil and Tillage Research, 82 (1), 57-64 (2005).

Perret J., S.O. Prasher, A. Kantzas y C. Langford; Three-Dimensional quantification of macropore networks in undisturbed soil cores, Soil Science Society of America Journal: 63, 1530-1543 (1999).

Perret J. y otros cuatro autores; Preferential solute flow in intact soil columns measured by SPECT scanning, Soil Science Society of America Journal: 64, 469-477 (2000).

Poulsen T.G. y otros cuatro autores; Spatial and temporal dynamics of air permeability in a constructed, agricultural field, Soil Science: 166, 153-162 (2001).

Ryan J., M. Singh y M.Pala; Long-Term Cereal-Based Rotation Trials in the Mediterranean Region: Implications for Cropping Sustainability, Advances in Agronomy: 97, 273-319 (2008).

Sarkar S. y S.R. Singh; Interactive effect of tillage depth and mulch on soil temperature, productivity and water use pattern of rainfed barley (Hordium vulgare L.), Soil and Tillage Research, 92 (1-2), 7986 (2007).

SSS, Soil Survey Staff; Soil Survey Manual, United States Department of Agriculture. Soil Conservation Service, Directive 430-V, Washington, USA (1981).

SPSS; Sigma Scan Pro 5.0 Image Analysis Software, SPSS Inc. Chicago, Illinois, USA (1999).

SPSS; Statistical Package for Social Sciences for Windows, Version 16. SPSS Inc. Chicago, Illinois, USA (2007).

Starr G.C.; Assessing temporal stability and spatial variability of soil water patterns with implications for precision water management, Agricultural Water Management: 72, 223-244 (2005).

Stephen N.N. y otros cuatro autores; Agro-hydrological evaluation of on-farm rainwater storage systems for supplemental irrigation in Laikipia district, Kenya, Agricultural Water Management: 73(1), 21-41 (2005).

Torquato S., C. Kim y D. Cule; Effective conductivity, dielectric constant, and diffusion coefficient of digitized composite media via first-passage-time equations, Journal of Applied Physic: 85, 1560-1571 (1999).

Vervoort R.W., S.M. Dabney y M.J.M. Romkens; Tillage and row position effects on water and solute infiltration characteristics, Soil Science Society of America Journal: 65, 1227-1234 (2001). 
Warner G.S., J.L. Nieber, I.D. Moore y R.A. Geise; Characterizing macropores in soils by computed tomography, Soil Science Society of America Journal: 53, 653-660 (1989).

Warkentin B.P.; Soil Structure: A History from Tilth to Habitat, Advances in Agronomy: 97, 239-272 (2008).

Weerts A.H., D. Kandhai, W. Bouten y P.M.A. Sloot; Tortuosity of na unsaturated sandy soil estimated using gas diffusion and bulk soil electrical conductivity: comparing analogy-based models and LatticeBoltzmann simulations, Soil Science Society of America Journal: 65, 1577-1584 (2001). 